Research paper

\title{
Lessons learnt from the implementation of same-day discharge after percutaneous coronary intervention
}

\author{
Yingyan Chen, $\mathrm{RN}^{\mathrm{a}}$, * \\ Andrea Marshall, RN, PhD ${ }^{\mathrm{a}, \mathrm{b}, \mathrm{c}}$ \\ Frances Lin, RN, PhD ${ }^{\mathrm{b}}$ \\ a School of Nursing and Midwifery, Griffith University, Gold Coast Campus, Southport, QLD 4215, Australia \\ ${ }^{\mathrm{b}}$ Menzies Health Institute, Queensland, School of Nursing and Midwifery, Griffith University, Australia \\ ${ }^{\mathrm{c}}$ Gold Coast Hospital and Health Service, Southport, Queensland, Australia
}

\section{A R T I C L E I N F O R M A T I O N}

\section{Article history:}

Received 10 April 2018

Received in revised form

2 October 2018

Accepted 17 November 2018

\section{Keywords:}

Cardiac catheterisation

Health resources

Patient discharge

Percutaneous coronary intervention

\begin{abstract}
A B S T R A C T
Background: Change in healthcare organisations is constant and requires adequate resources for effective implementation. Same-day discharge after percutaneous coronary intervention has been found to be no different from the patients who stayed overnight after procedure; however, its uptake remains low. Objectives: The aim of the study was to identify what factors helped or hindered the implementation of same-day discharge.

Methods: This interpretive study was conducted in a cardiac catheterisation suite of an Australian tertiary hospital between June and December 2016. Semistructured individual interviews with 26 healthcare professionals were conducted to explore the factors that influenced the implementation. A deductive approach to content analysis was guided by the theoretical domains framework.

Findings: Five domains, including beliefs about consequences, professional role and identity, resources, behaviour regulation, and optimism, were identified which were strongly related to the factors that impacted the implementation. The findings showed that participants believed same-day discharge benefits while also concerning about its safety and holding different opinions on eligibility criteria. The findings also showed that participants' involvement in the change process varied with no clear roles and responsibilities and that their understanding about same-day discharge evidence and the guideline also differed. Lack of dedicated resources was also identified as the hindrance to the implementation. Behaviour regulation domain depicted how communication was managed and how the care for same-day discharge patients was improved. Despite several issues identified, participants were optimistic with achievement.

Discussion and conclusion: This study has provided valuable insight into the factors that influenced the implementation, which will inform policymakers when designing interventions for future improvement. Plan for change and involvement of all stakeholders along with dedicated resources including time, people, and change management expertise are essential if hospitals want to see the success of change.

() 2018 Australian College of Critical Care Nurses Ltd. Published by Elsevier Ltd. All rights reserved.
\end{abstract}

\section{Introduction}

Change has become more critical than ever for healthcare organisations to improve efficiency, productivity, and service quality ${ }^{1}$ and to ensure the translation of evidence into clinical practice. ${ }^{2}$ While ongoing change is inevitable, many change initiatives can be unsuccessful. ${ }^{3}$ Key strategies in successful change

\footnotetext{
* Corresponding author.

E-mail address: yingyan.chen@griffithuni.edu.au (Y. Chen).
}

management are effective planning and engagement of staff in the change process, effective communication about the need for change, and implementation of strategies to sustain improvements over time. ${ }^{4}$ In many settings, a lack of dedicated resources and change management expertise in health care may contribute to ineffective change. ${ }^{5}$ This article focuses on how a health service approached change and provides lessons on what could or should be done differently to increase the likelihood of change success.

Models of care, which are broadly the way in which health services are delivered, are often changed to achieve efficiencies in 
healthcare delivery. For example, discharging surgical patients on their operative day is one strategy introduced to decrease the length of stay and associated healthcare costs. ${ }^{6}$ In the area of cardiac care, the increasing uptake of percutaneous coronary intervention (PCI), a procedure to treat obstructive coronary heart disease, has contributed to growing demands for cardiac services and acute hospital beds in recent years. ${ }^{7-9}$ Patients who undergo nonurgent $\mathrm{PCI}$ have traditionally been kept in hospital overnight for observation. However, a large body of research evidence has demonstrated that same-day discharge (SDD) after PCI (hereafter "SDD") is as safe as overnight stay. ${ }^{7}$ SDD also enhances hospital resources efficiency, ${ }^{8}$ improves cost-effectiveness, and increases patient satisfaction. ${ }^{9}$ Despite this, implementation of SDD remains variable worldwide. ${ }^{10}$ While the evidence for the safety and effectiveness of SDD is well described in the literature, there is little reported which can be used to help guide the implementation of SDD within complex healthcare systems. ${ }^{11}$

\section{Aim of the study}

The aim of the study was to identify factors that helped or hindered the implementation of SDD.

\section{Method}

\subsection{Study design and theoretical framework}

This interpretative study reports the qualitative component of a larger process evaluation study. A process evaluation, incorporating both quantitative and qualitative approaches, was conducted parallel to the hospital-led implementation of SDD. Quantitative process evaluation data, including patient selection, the SDD guideline compliance, and patients/relatives' response to the SDD process, have been analysed and reported elsewhere. ${ }^{12}$ SDD eligibility criteria were assessed at three time points: (1) in the preadmission clinic; (2) during the procedure; and (3) in the short-stay unit; SDD eligibility screening process is presented in Figure 1. A full description of the SDD guideline is available as Supplemental Material.

The theoretical domains framework (TDF) was used to guide this interpretive study. ${ }^{13}$ The TDF comprises 14 theoretical domains that represent a range of possible theory-based factors that influence health professional behaviour. ${ }^{13}$ As a theoretical lens, the TDF supports the assessment of cognitive, affective, social, and environmental factors which may influence behaviour. ${ }^{14}$ The TDF has

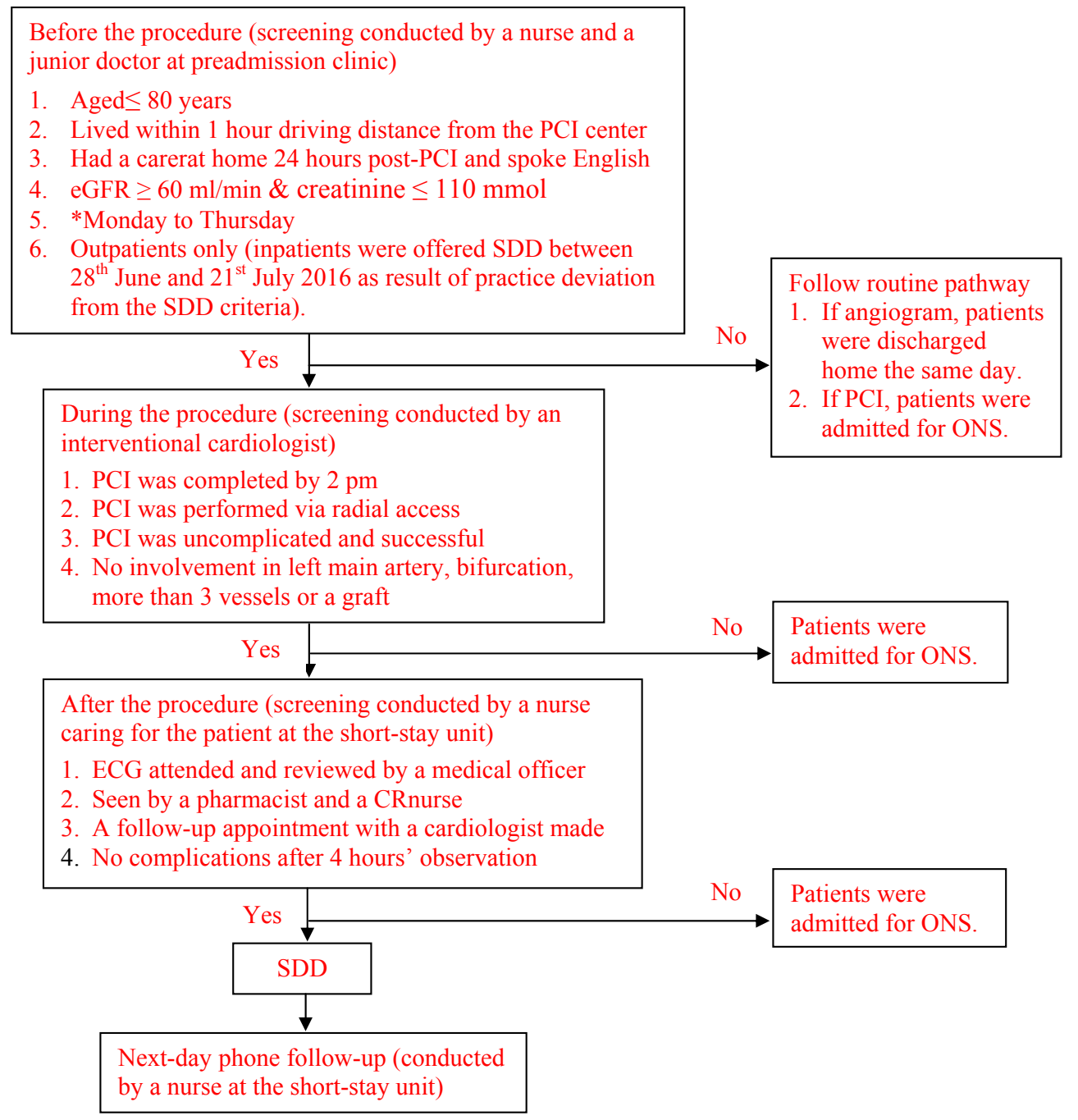

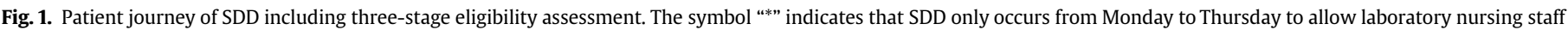

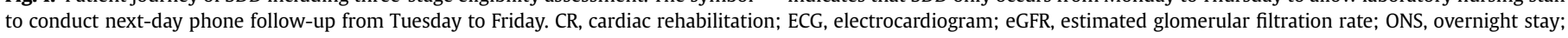
$\mathrm{PCI}$, percutaneous coronary intervention; SDD, same-day discharge. 
been used extensively in implementation research, both to prospectively facilitate implementation of healthcare interventions and retrospectively as a theory to guide a process evaluation. ${ }^{15}$ In this study, the TDF was used as an analytical framework.

\subsection{Setting and study context}

The study was conducted in a cardiac catheterisation suite of a 750-bed Australian tertiary hospital. The decision to implement SDD was made after a review of the cardiac services in 2014, 9 months after the relocation to a new hospital built on a Greenfield site. The implementation was initially planned for May 2015 but was delayed because of organisational constraints until June 2016. The timeline for SDD implementation is shown in Figure 2. Implementation of SDD was led by a registered nurse who held a leadership position within cardiac services, and implementation was incorporated into existing workloads. During the six-month implementation (June-December 2016), a total of 308 patients underwent PCI, of which 22 were sent home the same day. There were three instances in which inpatients undergoing PCI were discharged the same day, representing a deviation in the SDD criteria.

\subsection{Participants and recruitment}

All healthcare professionals (HCPs) who worked in the laboratory and who provided care to SDD patients were observed during the implementation. These included nurses and junior doctors who assessed patient eligibility before the procedure at the preadmission clinic, cardiologists and advanced trainees who were involved in managing SDD patients, nurses at the short-stay unit who provided direct patient care, cardiac rehabilitation nurses who provided cardiac rehabilitation information, and pharmacists who offered medication reconciliation, as well as the senior nurse who led the SDD implementation.

All HCPs who were employed by the cardiac services of the hospital were eligible to be invited for interviews. To capture a broad range of sources of information from key and relevant stakeholders, a purposive sampling strategy was chosen. ${ }^{16}$ There was no projected sample size for this study, rather based on the needed information being obtained. ${ }^{17}$ Initial interviews were with cardiologists and senior nurses, and final interviews were with cardiologists, senior nurses, and bedside nurses. A range of methods were used to recruit HCPs including a flyer visible in a staff room and doctors' office, email, and communication during ward meetings. Informed consent was obtained from all HCPs who participated in the study.

\subsection{Data collection}

Qualitative data collection was used to investigate factors that influenced the SDD implementation. The researcher observed implementation in practice and interactions among staff at the preadmission clinic and the short-stay unit, generating field notes during the observation period. Data were collected from 0800 to 1700 Monday to Thursday between June and December 2016 with an approximate $900 \mathrm{~h}$.

Individual interviews with HCPs were conducted at two time points: during the initial three months of implementation and at the end of the implementation. During the initial interviews, the focus was on developing an understanding of what HCPs understood SDD to be and of the evidence for this model of care. At this time, interview questions were also designed to understand the reasons for implementation delay. Interviews conducted at the end of the implementation were designed to explore HCPs' perceptions of factors that influenced the implementation. Semistructured interview guides were developed based on the $\mathrm{TDF}^{13}$ and tested before data collection. A contact summary was written immediately after each interview to enhance the data accuracy. Interviews lasted between 15 and 40 min. All interviews were audio-recorded and transcribed verbatim.

\subsection{Data analysis}

Data analysis commenced during data collection, which is a feature of qualitative data analysis. ${ }^{18}$ Interviews continued until data saturation was reached. ${ }^{19}$ The analytic method involved an iterative process of data analysis, in which a deductive content analysis was conducted to prepare, organise, and report findings relative to the factors that influenced the implementation. ${ }^{20}$ Nvivo (QSR version 11) was used to manage data analysis. ${ }^{21}$ The researcher first listened to the audio-recordings several times and read and reread interview transcripts and field notes line by line until she had become familiar with and immersed in the data. A categorisation matrix was then developed using the TDF domains as a coding framework, and data were then coded according to the

The guideline approval in process

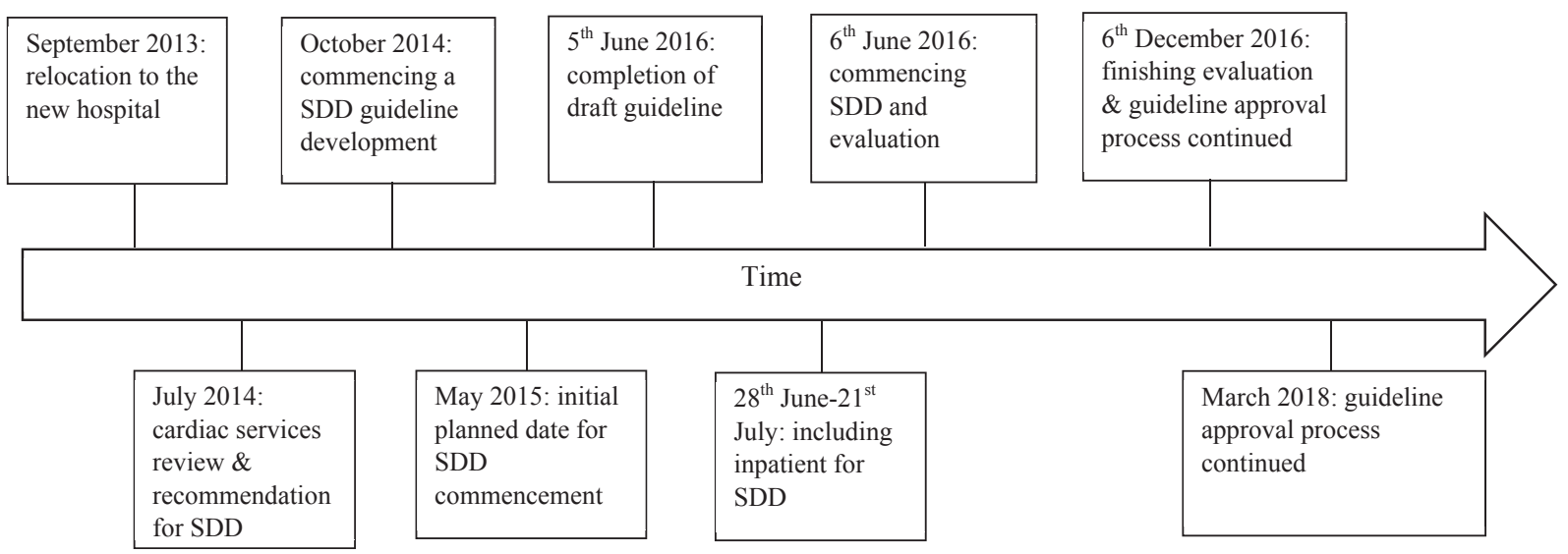

Fig. 2. Timeline of same-day discharge implementation. SDD, same-day discharge. 
domains. ${ }^{13}$ Categories were generated by grouping similar content within each domain. The data analysis results were discussed among all research team members several times, and revisions to codes, categories, and domains were undertaken. Any disagreement was resolved by developing a consensus.

\subsection{Rigor}

Credibility, confirmability, transferability, and dependability were ensured in this study to achieve trustworthiness for a qualitative inquiry. ${ }^{22}$ All sources of data (interview transcripts, contact summary, and field notes) were triangulated during the analysis to arrive at the codes, categories, and domains to demonstrate credibility. Throughout the study, the researcher remained reflexive to maintain confirmability by making notes of own personal thoughts and experience during data collection and analysis process. This study is not intended for generalisation; however, a comprehensive data set was obtained over a six-month period which contributed to the development of a rich and thick description in understanding the implementation and contributing to increased transferability. ${ }^{23}$ Dependability was enhanced by the researcher maintaining an audit trail of the research process, decisions, and analysis.

\subsection{Ethics}

The study was approved by the human research ethics committees (HREC) of the hospital (HREC/15/QGC/180) and the university (NRS/48/15/HREC).

\section{Findings}

Approximately 50 HCPs were observed during the six-month implementation period. A total of 26 HCPs participated in interviews, of whom eight were interviewed twice. Most participants were males. More than half were nurses, and the rest were cardiologists. Characteristics of participant demographics are shown in Table 1. Five domains were identified that predominantly related to the factors that influenced the implementation. These domains included beliefs about consequences, professional role and identity, resources, behavioural regulation, and optimism.

\subsection{Beliefs about consequences}

All participants demonstrated a strong understanding of the potential SDD benefits. Benefits to the patient were described as being able to go home earlier, fewer hospital adverse events, and increased patient satisfaction. For the organisation level, identified benefits included decreased hospital length of stay and improved use of inpatient beds, economic benefits, and alignment of service delivery with current best evidence.

Although all participants identified benefits to SDD, there was variability in the expressed beliefs about SDD safety. Most

Table 1

Demographic characteristics of healthcare professionals interviewed.

\begin{tabular}{ll}
\hline Categories & Total, $\mathrm{n}=26$ \\
\hline Age (mean, SD) & $48(12.6)$ \\
Male (n, \%) & $16(61.5)$ \\
Full-time employed (n, \%) & $19(73.1)$ \\
Clinical role & \\
$\quad$ Nursing team (n, \%) & $14(53.8)$ \\
$\quad$ Cardiologists (n, \%) & $12(46.2 \%)$ \\
Experience in the hospital (years) (median, IQR) & $9(3.0-16.0)$ \\
Experience in the CCS (years) (mean, SD) & $10(8.2)$ \\
\hline
\end{tabular}

CCS, cardiac catheterisation suite; IQR, interquartile range; SD, standard deviation. participants stated that SDD was safe if patients were fully assessed against the SDD criteria before discharge (Figure 1), whereas some (mainly cardiologists) expressed concerns about potential complications after SDD. For instance, one cardiologist commented that "They [patients] are going to be deprived of the care they may need in the immediate after putting in a stent, and the risk of hyperactive stent thrombosis is rare event but can occur in the first $24 \mathrm{~h}$ after stent implantation" (ID 6).

Opinions of eligibility criteria for SDD in the guideline also differed. Most participants commented that many eligibility criteria were conservative and stated that modifications should be made to accommodate more patients and improve the overall use of SDD. One of the identified criteria for change was the estimated glomerular filtration rate (eGFR), which nearly all participants agreed could be lowered down to 50,40 , or even $30 \mathrm{ml} / \mathrm{min}$ instead of current criterion of $\geq 60 \mathrm{ml} / \mathrm{min}$. Their reasoning for this was that contrast-induced renal injury usually occurs two to three days after the procedure; therefore, there was little, if any, benefit to keeping patients overnight. However, some participants commented that an eGFR $<60 \mathrm{ml} / \mathrm{min}$ was by definition stage-three chronic kidney disease and saw these patients at an increased risk. On the other hand, there was a lack of agreement among cardiologists about allowing outpatients who received stents to more than one vessel to be sent home the same day. This lack of agreement was perhaps based on differences in the risk perception of each cardiologist. Several nurses also commented that it was unsafe to send patients home the same day after receiving stents to more than one vessel, and their view may have been influenced by existing practice which was to admit patients to the coronary care unit after receiving stents to more than one vessel.

\subsection{Professional role and identity}

Although all participants in leadership positions recognised the importance of involving all staff in the change process, the practices of involving staff varied. Before the implementation, only a few cardiologists, senior nurses, and pharmacists were involved in the process of the guideline development, whereas many staff, including several cardiologists who had prior SDD experience and bedside nurses who delivered direct patient care, were not. Staff with no involvement were neither given an opportunity to provide input into the guideline development nor provided with an explanation of the reason for the change.

During the implementation, there were no clear roles and responsibilities defined among nursing and medical staff. On the one hand, cardiologists had an opportunity to discern if patients were eligible for SDD before the procedure; however, they often needed nurses to remind them of patients' eligibility. For instance, one cardiologist commented that "I think that perhaps the cardiologist and the advance trainee on the day of their procedure needs to be consulted more about whether the patient is going to be a SDD because patients are flagged as SDD but often we [medical staff] don't know until the procedure is over and then somebody comes and say "can this patient now go home" (ID 30). On the other hand, nursing staff perceived it might be their responsibility to take an active role because no one else enacted actively. Nurses expressed that "We need to be more proactive about it [driving SDD] and remind the doctors [if the patient can go home the same day]" (ID 16).

Moreover, there was a tension between nurses in the laboratory and in the inpatient ward when including inpatients for SDD. Laboratory nurses stated that discharging inpatients for SDD was not part of their roles and believed it was a task for ward nurses. However, ward nurses disagreed. Consequently, no nurses from both sides took the task of discharging inpatient home the same 
day; as a result, the inclusion of inpatient for SDD was suspended after three weeks.

\subsection{Resources}

Lack of dedicated resources was observed or perceived as an influential factor that delayed and impacted the implementation. No dedicated resources, including human resources, were allocated for implementing SDD, and existing staff were required to incorporate SDD into their already-busy workload. The senior nurse who led the change claimed that managing change was something gained from the experience as people did this all the time in their roles. There was no replacement staff to lead the implementation when the senior nurse went on leave for five weeks, which hampered the implementation.

No administrative support was provided to those who were responsible for the implementation. The guideline was developed to inform that clinical practice had not been approved by the hospital when SDD commenced and the approval process continued during the implementation period. Hence, the guideline was not available to staff as a resource. Other documentation, including an SDD PCI discharge instruction form, to be given to patients before discharge, was also not available when the implementation started.

\subsection{Behavioural regulation}

There was variability in participants' understanding about SDD and its evidence base. Several cardiologists and senior nurses understood SDD to be safe but were less certain about the strength of evidence underpinning SDD safety. Some cardiologists were unfamiliar with any evidence for SDD, whereas nurses described their understanding of SDD from personal experience or from colleagues at other hospitals.

At the beginning of the implementation, many participants were unaware of SDD implementation in this hospital, which was attributed to a lack of a planned communication strategy. The senior nurse responsible for managing the change found it challenging to ensure that all staff were made aware of the change to the model of care, as stated that "It was difficult in making sure all the staffs were on the same page and doing the right thing and doing the same thing" (ID 21). Communication was made difficult because staff did not regularly access work email, the mechanism through which most information was distributed. Consequently, when asked if participants were informed of the SDD implementation in this hospital during the implementation, several participants commented that "When we first started, we didn't know if it [SDD] started or not" (ID 15) and "Everything came into action one day" (ID 23).

Initial implementation of the SDD guideline was also challenging because there were inconsistencies in staff understanding of the criteria and recommendations. For those who were involved in the guideline development, their understanding of the process and criteria was much better than those who had no involvement. For those to whom the guideline was unfamiliar, they often felt confused and needed to frequently seek clarification about the eligibility criteria and asked questions such as "how many stents get put in the vessel [and still eligible for SDD]" (ID 31). Many staff attributed the confusion to lack of education sessions as shown in this quote "[SDD] not as defined or not everyone was educated to complete definitive of who does go home and who doesn't" (ID 31).

Despite challenges observed, nurses' enactment in the SDD implementation improved over time. At the beginning of the implementation, team leaders who scheduled laboratory lists did not give priority to SDD patients for procedures, leading to the patients being put last on the list, which meant the patients were automatically ineligible because the procedure completion time was outside what was permitted within the guideline. In addition, the information about the patients who were potential SDD candidates was not always known to all team members in the laboratory. However, since a nursing SCRUM (10-min daily huddle) was introduced by the senior nurse in the middle of the implementation, an SDD PCI checklist form was placed into the chart as a reminder for nurses to assess patients' eligibility. Gradually, nurses became more aware of which patients were eligible for SDD and started to highlight SDD patients on the white board to enable effective communication and to prioritise SDD patients.

\subsection{Optimism}

Although several issues encountered, participants held positive attitudes towards the SDD implementation. Positive comments were such as "We [nursing staff] have been waiting for this [SDD] for a long time" (ID 17), "It's nice to see it coming forward" (ID 29), and "There's absolutely no reason why this [SDD] should not work" (ID 14).

Participants also felt optimistic at the end of the implementation when interviewed. One participant stated optimistically that "We [our hospital] could set an example [of doing SDD] for the whole Queensland or .. we could be up there championing the cost for sameday PCI discharge" (ID 16). When asked to comment how SDD was implemented, participants gave the responses such as "successful" (ID 30), "probably eight out of ten" (ID 14), "it was very well" (ID 17), and "it's been implemented the way a new change is implemented, slowly but surely" (ID 29).

\section{Discussion}

Change is a common occurrence in healthcare organisations. ${ }^{24}$ However, how to best implement complex organisational change such as SDD remains a challenge. In a recent research study, ${ }^{25} 10$ primary barriers were identified to successful hospital change, some of which included poor implementation planning, failure to create buy-in and ownership of the change, ineffective leadership, suboptimal communication, unclear roles, and lack of dedicated resources and support. The key findings of our study strongly resonate with the findings ${ }^{25}$ and have provided additional details on lack of agreement in change eligibility criteria, variability in understanding of the change, its evidence base and the new model of care being implemented, variant participant involvement in the change process, ambiguous roles and responsibilities, and inadequate support in the form of dedicated resources.

In many clinical contexts, change is often being implemented too quickly without a thorough plan. ${ }^{25,26}$ The use of change theories/models could offer theoretical assumptions about the steps that clinicians must take to achieve the intended outcomes ${ }^{27}$ which contribute to long-term implementation success. ${ }^{28}$ There are a plethora of change theories/models including Roger's diffusion of innovation, ${ }^{29}$ Lewin's three-stage theory, ${ }^{30}$ and Kotter's change management theory ${ }^{31}$ described in literature. A common systematic three-stage change process can be followed which comprises preparing for change, implementing change, and evaluating and sustaining change, ${ }^{24}$ with each stage typically involving a series of smaller interrelated steps to help build personal engagement and commitment of staff members. ${ }^{24}$ Ignoring these steps creates the illusion of speed but often leads to undesirable outcomes. ${ }^{32}$ In our study, a theory or model of change was not used to inform the implementation, rather the implementation process was informed based on what clinicians' thought were reasonable approaches to change management. The organisation was not ready even when SDD commenced as the 
guideline approval was not obtained. Considering the limited resources and imperative need for change to be done rapidly within the health service, it is imperative that sufficient time and resources are provided so that clinicians who lead change will take the time needed to plan for change because "any change worth making is worth making right". ${ }^{25}$

Creating buy-in for the change is critical to ensuring effective implementation. ${ }^{25,33}$ When an organisation decides to implement change, it is important that all stakeholders are involved in the change process and through change management issues as this affects their job performance and active role within the organisation. ${ }^{34}$ Establishing stakeholder buy-in before the implementation enhances implementation outcomes because stakeholders who are engaged in the change process provide feedback on proposed change plans and listen to suggestions; ultimately they are more likely to be motivated and committed to the change. ${ }^{25,35}$ In our study, only selective staff in senior leadership roles were involved during the SDD guideline development process; most participants were not included in important discussions and decisions about how SDD might be implemented in this hospital. Lack of involvement in the change process from cardiologists might partially explain the reason of why they acted in a passive way during the implementation and why there was lack of agreement on SDD eligibility criteria. Therefore, involving staff, especially at the early stage, is vitally important in hopes of everyone being at the same page in the change process.

Establishing an effective communication strategy is another important component for successful organisational change. ${ }^{24,36}$ Helping staff to understand the need for change is vital, especially at the early stage of the process, as doing this will reduce uncertainty and improve transparency among team members. ${ }^{37}$ Explanation should also include the potential effects of future changes and how the changes will be implemented, as well as when different elements of the change implementation process will occur. ${ }^{38}$ In our study, most staff members were unclear about how SDD should be implemented due to the lack of communication on the implementation. There was no clear communication channel for staff to ask questions. This communication issue could be improved by establishing an effective communication plan with constant, two-way communication between those responsible for leading the change and those responsible for making the change happen to produce superior outcomes. ${ }^{25}$

Having adequate organisational support is essential for successful implementation of change. ${ }^{5,25}$ However, clinicians are often not well supported to manage change. It has been reported that nurse leaders who have the most demanding work schedules are often responsible for implementing change, ${ }^{39}$ and they often lack the requisite competencies and skills to meet the challenges ${ }^{40}$ as they receive little training that prepares them adequately to assume leadership responsibilities. ${ }^{41}$ Subsequently, most learn through trial and error or on-the-job training. ${ }^{42}$ Nurse leaders also may not have sufficient time and resources allocated to support the change process such as equipment, budget, and staffing, making it hard to achieve desirable outcomes. ${ }^{25}$ In our study, those responsible for the implementation "learned on the job". In addition, nurses undertook their individual roles of the implementation in addition to their existing busy workload with little dedicated time and resources allocated. There was neither a coleader nor a project manager assigned in this implementation to maintain implementation continuity. Consequently, no replacement of staff was available to cover the duty of the senior nurse who was absent for five weeks, leading to the interruption of the implementation. As a health service undergoes continual complex change, it is essential for change leaders to possess the knowledge, skills, and attributes required to effectively manage change and also to ensure that they are supported by dedicated resources and time. ${ }^{39}$
It is evident that an effective change is integral to improve quality, safety, and efficiency of health care. ${ }^{1,39}$ Having a detailed implementation plan, staff engagement, end-user input, strong communication, and adequate resources could have all contributed to successful implementation of SDD. More inclusive SDD criteria, which aligned with recommendations in the literature, may have also helped increase the number of patients eligible for SDD which, in turn, may have had a more positive impact on the length of hospital stay and healthcare costs. Therefore, it is crucial to optimise the change management process to produce tangible benefits to the health service.

\section{Strengths and limitations}

One of the strengths of this study is the use of an external team to evaluate the implementation, a necessary step to avoid bias. ${ }^{43}$ Two limitations of this study are acknowledged. First, there could have been social desirability bias in all interviews during which participants answered one of the questions "how well do you think SDD was implemented?" in a manner that was viewed as favourable by the researcher. Also, the study was conducted within one Australian tertiary hospital, and the findings may not necessarily be transferred to other settings in Australia and beyond. However, the implementation issues that arose and lessons learnt from this study are important for any clinician to consider when implementing change in other healthcare settings.

\section{Conclusion}

One of the main challenges in healthcare organisations is the effective change management. This study has taken the first step in responding to the call of identifying effective ways to implement SDD into routine clinical practice. While hospitals are often under increasing pressure to implement change in a short period of time with the limited resources, it is not surprising that clinicians leading change often fail to achieve positive outcomes. As "any change worth making is worth making right", plan for change with strong stakeholder buy-in coupled with dedicated resources including time, people, and change management expertise is essential in leading to a successful change.

\section{Authors' Contribution}

Yingyan Chen contributed to the data collection. All three authors contributed to the conception and design of the study; data analysis and interpretation of the data; drafted and revised the manuscript; approved the final version of the manuscript.

\section{Acknowledgements}

The authors would like to acknowledge the Griffith University Patricia Barlow Scholarship in providing support for conducting this research and the staff in the hospital for their participation in this study.

\section{Appendix A. Supplementary data}

Supplementary data to this article can be found online at https://doi.org/10.1016/j.aucc.2018.11.066.

\section{References}

[1] Stanford J. Breaking the cycle of failed change management. Englewood Cliffs, NJ, United States: American Society for Training \& Development; 2016. 
[2] Kristensen N, Nymann C, Konradsen H. Implementing research results in clinical practice: the experiences of healthcare professionals. BMC Health Serv Res 2016;16(1):48.

[3] Jacobs G, van Witteloostuijn A, Christe-Zeyse J. A theoretical framework of organizational change. J Organ Change Manag 2013;26(5):772-92.

[4] Hayes J. The theory and practice of change management. 4th ed. Houndmills, Basingstoke, Hampshire; New York, NY: Palgrave Macmillan; 2014.

[5] Al-Haddad S, Kotnour T. Integrating the organizational change literature: a model for successful change. J Organ Change Manag 2015;28(2):234-62.

[6] Oh J, Perlas A, Lau J, Gandhi R, Chan VW. Functional outcome and costeffectiveness of outpatient vs inpatient care for complex hind-foot and ankle surgery. A retrospective cohort study. J Clin Anesth 2016;35:20-5.

[7] Bundhun PK, Soogund MZ, Huang WQ. Same day discharge versus overnight stay in the hospital following percutaneous coronary intervention in patients with stable coronary artery disease: a systematic review and meta-analysis of randomized controlled trials. PloS One 2017;12(1), e0169807.

[8] Shroff A, Kupfer J, Gilchrist IC, Caputo R, Speiser B, Bertrand OF, et al. Sameday discharge after percutaneous coronary intervention: current perspectives and strategies for implementation. JAMA Cardiol 2016;1(2):216-23.

[9] Amin AP, Crimmins-Reda P, Miller S, Rahn B, Caruso M, Pierce A, et al. Novel patient-centered approach to facilitate same-day discharge in patients undergoing elective percutaneous coronary intervention. J Am Heart Assoc 2018;7(4).

[10] García-Izquierdo Jaén E, Goicolea Ruigómez FJ. Same-day discharge after elective percutaneous coronary intervention: a safe strategy, but for which patients? Rev Esp Cardiol 2017;70(7):524-6.

[11] Chen YY, Marshall A, Lin F. Implementation strategies for same day discharge post percutaneous coronary intervention: an integrative review. Worldviews Evidence-Based Nurs 2016;13(5):371-9.

[12] Chen YY, Lin F, Marshall A. Implementing same day discharge following percutaneous coronary intervetion: a process evaluation. J Nursing Care Qua 2019;34(1).

[13] Cane J, O'Connor D, Michie S. Validation of the theoretical domains framework for use in behaviour change and implementation research. Implement Sci $2012 ; 7: 37$

[14] Atkins L, Francis J, Islam R, Connor D O', Patey A, Ivers N, et al. A guide to using the Theoretical Domains Framework of behaviour change to investigate implementation problems. Implement Sci 2017;12:77-94.

[15] Phillips CJ, Marshall AP, Chaves NJ, Jankelowitz SK, Lin IB, Loy CT, et al. Experiences of using the Theoretical Domains Framework across diverse clinical environments: a qualitative study. J Multidiscip Healthc 2015:8:139-46.

[16] Liamputtong P. Qualitative research methods. 4th ed. South Melbourne: Oxford University Press; 2013.

[17] Bernard HR, ProQuest E. Research methods in anthropology: qualitative and quantitative approaches. 5th ed. Lanham, Md: AltaMira Press; 2011.

[18] Polit DF, Beck CT. Resource manual for nursing research: generating and assessing evidence for nursing practice. 9th ed. Philadelphia: Wolters Kluwer Lippincott Williams \& Wilkins; 2012.

[19] Glaser BG, Strauss AL. The discovery of grounded theory: strategies for qualitative research. 1st ed. Hawthorne, N.Y; Chicago: Aldine De Gruyter; 1967.

[20] Elo S, Kyngas H. The qualitative content analysis process. J Adv Nurs 2008;62(1):107-15.

[21] QSR International. NVivo qualitative data analysis software. Version 11. 2017
[22] Lincoln YS, Guba EG. Naturalistic inquiry. Beverly Hills, Calif.: Sage Publications; 1985.

[23] Creswell JW. Qualitative inquiry \& research design: choosing among five approaches. 3rd ed. Los Angeles: SAGE Publications; 2013.

[24] Allen B. Effective design, implementation and management of change in healthcare. Nurs Stand 2016;31(3):58-71.

[25] Longenecker CO, Longenecker PD. Why hospital improvement efforts fail: a view from the front line. J Healthc Manag 2014;59(2):147-57.

[26] Huq Z. Managing change: a barrier to TQM implementation in service industries. Manag Serv Qual: Int J 2005;15(5):452-69.

[27] Grol R, Bosch M, Hulscher M, Eccles M, Wensing M. Planning and studying improvement in patient care: the use of theoretical perspectives. Milbank Q 2007; 85(1):93-138.

[28] Mitchell G. Selecting the best theory to implement planned change. Nurs Manag Apr 2013;20(1):32-7.

[29] Rogers EM. Diffusion of innovation. New York; London: Free Press; 2003.

[30] Lewin K. Field theory in social science: selected theoretical papers. Westport, Conn.: Greenwood Press; 1975.

[31] Kotter JP. Leading change. Boston, Mass: Harvard Business School Press; 1996.

[32] Grol R, Wensing M. Effective implementation of change in healthcare: a systematic approach. In: Grol R, Wensing M, Davis D, editors. Improving patient care: the implementation of change in health care. 2nd ed. Chichester, UK: Hoboken, NJ, USA: Wiley Blackwell, BMJ/Books; 2013.

[33] Geerligs L, Rankin NM, Shepherd HL, Butow P. Hospital-based interventions: a systematic review of staff-reported barriers and facilitators to implementation processes. Implement Sci 2018;13(1):36.

[34] Lüscher LS, Lewis MW. Organizational change and managerial sensemaking: working through paradox. Acad Manag J 2008;51(2):221-40.

[35] Timmings C, Khan S, Moore JE, Marquez C, Pyka K, Straus SE. Ready, set, change! Development and usability testing of an online readiness for change decision support tool for healthcare organizations. BMC Med Inf Decis Making 2016;16(1):24.

[36] Ledger A, Edwards J, Morley M. A change management perspective on the introduction of music therapy to interprofessional teams. J Health Organisat Manag 2013;27(6):714-32.

[37] Van de Ven AH, Sun K. Breakdowns in implementing models of organization change. Acad Manag Perspect 2011;25(3):58-74.

[38] Allen J, Jimmieson NL, Bordia P, Irmer BE. Uncertainty during organizational change: managing perceptions through communication. J Change Manag 2007;7(2):187-210.

[39] Kumah E, Ankomah SE, Antwi F. The role of first-line managers in healthcare change management: a Ghanaian context. Int J Biosci Healthc Technol Manag 2016;6(3):20-33.

[40] Sherman RO, Schwarzkopf R, Kiger AJ. Charge nurse perspectives on frontline leadership in acute care environments. ISRN Nurs 2011;2011:1-8.

[41] Schell WJ, Kuntz SW. Driving change from the middle: an exploration of the complementary roles and leadership behaviors of clinical nurse leaders and engineers in healthcare process improvement. Eng Manag J 2013;25(4): 33-43.

[42] Phillips N, Byrne G. Enhancing frontline clinical leadership in an acute hospital trust. J Clin Nurs 2013;22(17-18):2625-35.

[43] Conley-Tyler M. A fundamental choice: internal or external evaluation? Eval J Australas 2005;4(1 \& 2):3-11. 